\title{
Urodimento
}

REVISTA DE ESTUDOS EM ARTES CÊNICAS

E-ISSN 2358.6958

\section{Del templo al escenario digital: La danza clásica india en tiempos pandémicos}

\author{
Miriam Lamas-Baiak
}

Para citar este artigo:

LAMAS-BAIAK, Miriam. Del templo al escenario digital: La danza clásica india en tiempos pandémicos. Urdimento - Revista de Estudos em Artes Cênicas, Florianópolis, v. 2, n. 41, set. 2021.

do) DOI: http:/dx.doi.org/10.5965/1414573102412021e0121

Este artigo passou pelo Plagiarism Detection Software| iThenticate 


\section{Del templo al escenario digital: La danza clásica india en tiempos pandémicos}

Miriam Lamas-Baiak ${ }^{1}$

\section{Resumen}

Este artículo trata de las acciones emprendidas por el medio digital, en relación a las danzas clásicas indias, en concreto, los estilos bharata natyam y kathak, como resultado de la crisis del COVID-19. Retrata los procesos desarrollados más allá de las clases remotas y de vídeos disponibles en internet, cómo, por ejemplo, a través de foros de debate, congresos, espacios para adquisición y distribución de contenidos e información. Finalmente, presenta la contribución de los medios digitales a la danza india en un contexto pandémico adverso, ampliando algunos territorios y delimitando otros ya existentes, abriendo todo un campo de investigación y creación que continúa a expandirse, creando un nuevo marco de producción y recepción para la danza india.

Palabras claves: Medio digital. Pandemia. Danza clásica india. Bharata natyam. Kathak.

\section{Do templo ao cenário digital: A dança clássica indiana em tempos pandêmicos}

\section{Resumo}

Este artigo trata das ações empreendidas por meio digital, em relação às danças clássicas indianas, em específico, os estilos bharata natyam e kathak, em decorrência da crise do COVID-19. Retrata os processos desenvolvidos para além das aulas remotas e dos vídeos disponibilizados na internet, como, por exemplo, através de fóruns de debate, congressos, espaços para aquisição e distribuição de conteúdos e informação. Por fim, apresenta a contribuição dos meios digitais à dança indiana em um contexto pandêmico adverso, ampliando alguns territórios e limitando outros já existentes, abrindo todo um campo de investigação e criação, que continua a se expandir, criando um novo marco de produção e recepção para a dança indiana.

Palavras-chave: Meio digital. Pandemia. Dança clássica indiana. Bharata natyam. Kathak.

Doutoranda em Humanidades: Linguagem e Cultura pela Universidad Rey Juan Carlos, mestrado em Artes Escénicas pela Universidad Rey Juan Carlos. Especialização em Fisiologia do Exercício pela PUC-PR. Graduação em Dança - Licenciatura e bacharelado - pela Faculdade de Artes do Paraná (atual UNESPAR). m.lamas.2018@alumnos.urjc.es 


\title{
From the temple to the digital scene: Indian classical dance in
} pandemic times

\begin{abstract}
This article addresses the actions taken through digital technologies in support to Indian classical dances, as a result of the COVID-19 crisis. In particular regarding the styles Bharata Natyam and Kathak. It presents the processes developed in addition to remote classes and videos that were made available on the internet; such as debate forums, congresses, spaces for the acquisition and distribution of content and information. Ultimately, it discuss the contribution of digital media to Indian dance in an adverse pandemic context. How it expands some territories and constricts existing ones; opens up a whole field of research and creative activity that continues to grow, resulting in a new framework for production and reception of the Indian dance.
\end{abstract}

Keywords: Digital media. Pandemic. Indian Classical dance. Bharata natyam. Kathak. 


\section{La danza clásica india}

La danza clásica india tiene una historia de siglos y miles de años, relacionada con la religión hindú, los textos védicos y los templos, se fue desarrollando en diversos estados del país, resultando actualmente en 8 estilos: bharata natyam, kathak, kathakali, kuchipudi, manipuri, mohiniyattam, odissi y sattriya. El bharata natyam y el kathak son los más conocidos y más practicados en todo el mundo, además, presentan más apertura a los cambios que las nuevas tecnologías están trayendo, por esto, fueron elegidos para este estudio². Estas danzas, a pesar de ser consideradas tradicionales, vienen evolucionando con el paso del tiempo, sufriendo diversas transformaciones, influenciadas por la cultura de otros países, por la colonización y occidentalización, por el movimiento de independencia de India, y en los días actuales por las nuevas tecnologías.

El bharata natyam, danza originaria del estado de Tamil Nadu, sur de India, nació en los templos hindúes con las danzarinas hereditarias. Estas mujeres, conocidas en general como devadasis, dedicaban su vida al servicio del templo, en el cual la danza estaba incluida formando parte del ritual diario. Su profesión era transmitida de generación a generación, pudiendo también el templo recibir donaciones de niñas. Ellas eran divididas en diversas categorías, y algunas podrían tener alto prestigio social, poseer riquezas y tierras. No se casaban con un hombre mientras prestaban sus servicios, pues estaban casadas con un dios, con el dios al que estaba dedicado el templo en el que trabajaban, pero esto no les prohibía tener relaciones sexuales ni era considerado inmoral. La danza que estaba dentro de los templos empezó a salir a las calles en las procesiones, después llegó a los palacios, donde en los siglos XVIII y XIX tuvo gran desarrollo, y posteriormente en el siglo XX con la influencia de la moral victoriana y con el movimiento nacionalista de independencia el sistema devadasi fue prohibido, la danza se extendió a los teatros con la alta casta india, los brahmanes, sufriendo cambios en su técnica y 
estructura (Kersenboom, 1987).

Rukmini Devi Arundale, es el nombre principal en la reforma del bharata natyam. Era brahmane, casada con un inglés, miembro de la Sociedad Teosófica, con contacto frecuente con occidente y sus costumbres. Hizo ballet clásico bajo la supervisión de Anna Pavlova, bailarina rusa, que la incentivó a rescatar la danza de su país, de esta forma, empezó la práctica del bharata natyam con más de 30 años de edad, fundando poco tiempo después su propia escuela, Kalakshetra, en 1936, que estaba vinculada a la Sociedad Teosófica, cambiando profundamente la técnica y la enseñanza de esta danza que fue mantenida durante cerca de 2 mil años por las devadasis (Meduri, 2008; Nachiappan, 2001).

En estos cambios tenemos una gran influencia del ballet clásico: en las líneas corporales; en la simetría; en la estructura de enseñanza en niveles, exámenes, certificados; en la creación de los dramas con la misma estructura de los repertorios de ballets; y en el uso del espejo en el aula. También se observa una fuerte influencia de occidente en la adaptación de esta danza templaria a los escenarios de los teatros, adoptando una estructura de espectáculo y no de ritual. Además, ella quitó los movimientos de cadera, que en la época eran vistos como inmorales, relacionados con la sexualidad, con la prostitución y con las devadasis (Baiak, 2019).

Esta danza reformada en el siglo pasado es vista y considerada tradicional por muchos indios y extranjeros, como si no hubiera tenido cambios. Actualmente se está intentando concienciar a las personas sobre la historia y el proceso de desarrollo, pretende además recuperar la danza como era antes de la reforma. Nrithya Pillai ${ }^{3}$, una descendiente de las devadasis, busca además rescatar el valor de su casta, la importancia de sus antecedentes y su lugar en la historia actual del bharata natyam.

El kathak, danza del norte de India, del estado de Uttar Pradesh, que también abarca la región del cinturón del Ganges, con los estados de Rajasthan, Bihar,

3 Nieta del reconocido nattuvanar (músico) Guru Swamimalai K. Rajarathnam Pillai de la casta isaivellalar (comunidad de las danzarinas hereditarias que danzaban sadir, la danza que precedió el bharata natyam). Bailarina y profesora, ella es una voz activa en India y en las redes sociales. 
Madhya Pradesh, Gujarat, Maharastra y Punjab. Su historia tradicional dice que kathak deriva de katha, que significa historia, donde los kathaks, contaban historias a través de la danza. Estos hombres serían de la casta de los brahmanes, teniendo registros de su existencia en el texto épico hindú Mahabharata y en otros textos sánscritos (Kothari, 1989; Narayan, 2001, 2004 y 2005). Pero nuevas investigaciones y teorías, como la de Walker (2004, 2009, 2010a y 2010b) demuestran que el kathak fue desarrollado por las mujeres en los palacios del norte del país.

Las investigaciones de la autora apuntan que los kathaks eran más bien un grupo de sátiros que de bailarines, actuando dentro de lo que llamamos teatro más que en danza 4 . Siendo el kathak desarrollado, probablemente, con las tawaifs o tavaifs, mujeres que danzaban en las cortes, influenciadas, además de otras civilizaciones, por la danza y cultura persa. Al contrario que las devadasis que eran mujeres dedicadas al templo, las tawaifs eran mujeres a servicio del palacio, que fueron más tarde relacionadas con la prostitución así como las primeras. La desaparición de su papel en la danza posiblemente se debe a esta relación, el movimiento nacionalista en el norte del país retiró las mujeres de la historia.

La danza que conocemos hoy también es resultado de una reforma hecha en el siglo pasado, convirtiendo la danza de la corte en una danza profesional, influenciada también por occidente, donde encontramos indios inspirados en bailarines occidentales como la americana St. Denis y nuevamente influenciados por Anna Pavlova. Según Skiba (2016) el kathak tiene una cultura híbrida que sigue por variados caminos. Para Walker (2004 y 2009) es fruto de una fusión cultural, es devocional y de entretenimiento, es masculina y femenina, es hindú y musulmana, es una danza contemporánea con raíces milenarias.

\section{El escenario digital}

Estos dos estilos de danza clásica, con fuertes raíces y grandes mutaciones, viven actualmente en un mundo globalizado e influenciado por las nuevas tecnologías, estando presentes en el mundo físico y virtual, este último, según

${ }^{4}$ En India no había una diferencia entre danza y teatro, ambas eran relacionadas con la palabra natya o natyam, que puede ser traducida como drama. (Muni, 2006) 
Merejo (2017), es el territorio que se está expandiendo en el mundo. Desde el siglo $X X$ las nuevas tecnologías vienen determinando la realidad cultural, influenciando fuertemente la percepción del público, en variados puntos de vista como también afectando a las formas de construcción del creador, dando un nuevo lugar a las artes escénicas, un lugar mediático (Bernal, 2008). Lo que ya venía ganando espacio en los últimos años tuvo un gran avance con la pandemia del COVID-19. La única forma de supervivencia fue adherirse al medio digital, a las redes sociales y a las plataformas de vídeos, cambiando los procesos de creación, recepción y enseñanza. Limitando por un lado pero aumentando límites por otro. Al final la internet separa al mismo tiempo que une, distancia al mismo tiempo que aproxima, y abre nuevas puertas donde otras fueron cerradas y viceversa, ampliando territorios y delimitando otros.

Tomando ejemplo de la propia pandemia, internet separó físicamente alumnos y profesores, bailarines y público pero los unió en el medio digital. Los distanció pero aproximó a otros que viven en ciudades, países y continentes diferentes. La pandemia cerró aulas, escuelas y teatros pero abrió una nueva puerta, ni peor ni mejor, una puerta diferente que posibilitó la continuidad de la danza en un escenario digital. Y este nuevo mercado digital que está creciendo cada día hace que puertas físicas cierren definitivamente, pues los hábitos van cambiando y la comodidad de internet pasa a ser preferida por muchos usuarios.

Según López (2018) las tecnologías de información y comunicación (TIC) son herramientas indispensables en los escenarios de comunicación e interacción. Para Lachino (2017) no debemos pensar en estas tecnologías como una relación entre los aparatos y las coreografías sino entenderlas como una nueva forma de interacción que da nuevas posibilidades. De esta forma es como interactuamos entre la danza y el mundo virtual que va a traer nuevos procesos de creación, de producción, de enseñanza y de recepción, es actuar de diferentes maneras que van a hacer de la tecnología un cambio para la danza, si no, será solamente la danza de siempre insertada en el medio virtual. Esto es, la interacción significa cambiar la manera en que ocurre la danza, en consecuencia, también cambiará la forma en que esta danza será recibida.

Escuelas, instituciones, compañías, grupos, bailarines, profesores pasaron a 
promover eventos, festivales, espectáculos, coreografías, clases, debates, en modo virtual. El abanico de posibilidades y oferta creció ilimitadamente desde marzo de 2020, muchas veces de forma gratuita. Según Tredezini ${ }^{5}$ (2020) la tecnología pasó a ser un soporte para la danza, ampliando sus límites en el modo virtual (desmaterialización), llegando a personas en todo el mundo que tengan una conexión con internet (democratización), con coste reducido o cero (desmonetización), creando así un nuevo negocio (disrupción), conocidos como los 4 D's.

Según Mattos $^{6}$ (2021) estos 4 D’s están dentro de los 6 D’s Exponencial, que son las etapas del crecimiento de una tecnología, desarrollados por Peter Diamantis ${ }^{7}$. Aplicados a la danza, ella ve el 1 D - digitalización, cuando la danza es transformada en códigos en internet, siendo reproducida y compartida, además de multiplicar su difusión, la convierte en perpetua, sin depender del profesional. El 2 D - decepción, es el crecimiento lento del resultado, ya que los números se van duplicando en números pequeños hasta llegar en un exponencial grande, siendo necesaria la persistencia del profesional para pasar por esta etapa. El 3 D disrupción, es un rompimiento, la danza cambia de ambiente, está en un nuevo escenario, que es digital. En el 4 D - desmonetización, la tecnología convierte un producto o un servicio en algo gratuito o con coste reducido pero es necesario tener una estrategia para enseguida ofrecer algo monetizado, la desmonetización sería un cebo para llegar a más gente. Desmonetizamos el teatro, entregamos el arte gratuitamente pero debemos crear un nuevo mercado monetizado. En el 5 D

${ }^{5}$ Bárbara Tredezini es fundadora del festival online e internacional de vídeodanza Festival Videodance. Es especialista en Marketing Digital por la Universidad del Valle del Silicio en Estados Unidos (Udacity), con post grado en Transformación digital e innovación por la BBI de Chicago en concertación con el Centro Universitario Celso Lisboa, y grado en Danza por la Facultad de Artes del Paraná, actual UNESPAR. Actúa desde 2007 en las áreas de design gráfico y webdesign.

https://videodance.com.br/

https://www.facebook.com/videodancefestival

https://www.instagram.com/videodancefestival/

6 Flávia Mattos es formada en Danza por la Facultad de Artes del Paraná, actual UNESPAR, actuando profesionalmente a 22 años en la área artística y emprendedora. A través de su programa Minha Arte Lucrativa ayuda los profesionales de danza a monetizaren su trabajo en el medio digital.

https://flaviacafe.com.br/

https://www.facebook.com/flavia.mattos.391

https://www.instagram.com/flaviacafe/?hl=pt

Peter Diamandis, engeniero y medico, fundó varias empresas en la área de espacio, longevidad, educación y capital de riesgo, siendo un visionario en la área de tecnología. https://www.diamandis.com/ 
- desmaterialización, el producto dejaría de ser material pero la danza ya es algo que no es material, como un cuadro por ejemplo, sin embargo, ella no deja de existir, y en internet puede ser materializada y eternizada, adaptándola a las plataformas online y al consumo digital. En el 6D - democratización, la danza es accesible a todos por medio de la tecnología.

La autora cree que la crisis del Covid-19 hizo que el artista se conectase más a una realidad práctica, rompiendo creencias limitantes del mercado, aumentando posibilidades, descubriendo nuevas formas de producir, adaptándose e innovando, cambiando las herramientas, creando estrategias, metodologías y dinámicas específicas, e identificando las motivaciones de un consumidor digital para que este se mantenga involucrado en el producto o servicio ofrecido, dando un nuevo valor al mercado de la danza.

\section{Las clases online}

Dentro de este mundo de posibilidades encontramos la danza insertada en el mundo virtual e interactuando con él, con diversos grados de compromiso y calidad, cambiando la forma de ser, estar y relacionarse en el mundo o no. Así como otros estilos de danza, tenemos el bharata natyam y el kathak actuando y relacionando en estos diferentes grados. Diversas acciones fueron y están siendo realizadas en esta época de pandemia. Podemos observar en todo el mundo clases online como único medio para la enseñanza, tanto instituciones como profesores autónomos impartiendo clases a través de plataformas gratuitas y pagas, como Zoom, Jitsi Meet, Google Meet, Microsoft Teams, entre otras. Según Tavares (2019) las innovaciones en educación se iniciaron en 1960, y las tecnologías de información estarían relacionadas con el desarrollo económico, siendo una estrategia y una propuesta pedagógica. De esta forma, la pandemia hizo de las clases virtuales la única estrategia para que estas ocurriesen, y dentro de esta estrategia varias propuestas empezaron a ser formuladas y están aún en desarrollo, reflejándose directamente en la economía, tanto de las empresas tecnológicas cuanto de los docentes y alumnos.

La enseñanza de la danza cambió para el medio digital por necesidad, 
profesores, alumnos y escuelas no estaban preparados para esta modificación, no hubo tiempo para pensar y planear el cómo una clase práctica de danza podría implantarse en el modo online. Después de un año del inicio de la pandemia aún encontramos problemas en esta transición. Pero Mattos (2021) siente un avance en el mercado, donde el profesional de la danza está aprendiendo a vender su producto y a emprender en el mundo digital, aumentando sus referencias y las opciones del consumidor. Recordando a Lachino (2017) danza y tecnología necesitan interactuar y cambiar, es necesario crear nuevas metodologías. Por ejemplo, la danza india está llena de detalles de ojos, manos y pies, que en una visión general en una pantalla no es posible verlos, la visibilidad es limitada, tanto alumno como profesor necesitan aproximarse a la cámara y enseñar solamente una parte del cuerpo para que sea vista, en una pantalla de móvil la situación es aún peor. También es necesario, muchas veces, ver alumno por alumno en la pantalla, ya que la visión general no permite una buena visibilidad para el profesor. Hay aparatos que reflejan la imagen, otros no, así es difícil saber cuándo es derecha y cuándo es izquierda. Hay también retraso en el sonido, y en estas danzas en que los pies hacen el ritmo de la música, dificulta saber si el ejecutante está en un ritmo incorrecto o es por el retraso de internet, siendo necesario grabar un vídeo para que imagen y sonido estén en sincronía y así enviarlo al profesor y/o alumno. El espacio también perjudica, pues no es ideal, es el cuarto, el salón, la cocina que no tienen un suelo adecuado para la danza, muchas veces es pequeño y habiendo otras personas compartiendo el mismo lugar. Quien vive en apartamento no puede poner el sonido alto ni hacer los golpes con los pies de la manera que deben ser hechos por el ruido y por la vibración. La democratización, citada por Tredezini (2020) y Mattos (2021) termina de esta manera por no ser tan democrática ya que quien puede pagar por una plataforma digital y con una buena conexión a internet tendrá más herramientas y más calidad en la transmisión, y quien posee un espacio más adecuado y amplio tendrá una mejor práctica.

Además, por todas estas dificultades, el alumno necesita de más medios para el aprendizaje, como disponibilidad de vídeos en drives, links, correos electrónicos, etc., material didáctico y teórico para apoyo, grupos y mensajes privados en WhatsApp para dudas e información. Teniendo el profesor que disponer más 
tiempo para su docencia, creando nuevas metodologías para conseguir transmitir por el mundo virtual lo que antes era transmitido por el mundo físico. Pues la danza que en la presencia física es tridimensional en el digital se convierte en bidimensional, la percepción cambia, la pantalla limita el grado de percepción de movimiento tanto del profesor como del alumno. Además no hay tacto, que es muy importante en las correcciones corporales, donde el alumno sólo consigue comprender un movimiento a través de este sentido y no de una explicación oral.

Esta dificultad de la enseñanza bidimensional ocurre principalmente en niños y alumnos de iniciación, ya que estos no tienen una conciencia corporal desarrollada. Es muy diferente impartir una clase online para un profesional de la danza, que para un estudiante, el grado de comprensión de ellos no es el mismo, y la explicación del profesor no va llegar de la misma manera, está faltando un componente en el proceso de enseñanza que es la percepción visual presente/física y táctil.

Algunas escuelas y profesores también optaron en el inicio de la pandemia por poner a disposición del alumno clases con vídeos grabados. Esta metodología es más limitante, ya que no existe una interacción entre profesor y alumno, hay sólo una transmisión de información que no sabemos cómo llega al alumno ni sabemos cómo éste ejecuta la clase, si hace los movimientos de manera correcta o no.

Tomando como ejemplo mi propia experiencia como profesora de danza india, las clases online me resultan un desafío. Vivo en un apartamento compartido y necesito del salón porque en mi cuarto no hay espacio, el wifi no llega al piso, tengo que utilizar la conexión a internet de datos del móvil, uso plataforma gratuita con menos opciones de herramientas, no puedo golpear los pies, no puedo poner la música alta. Cuando la escuela donde trabajaba pedía que las clases fueran a través de vídeos grabados, me llevaba cerca de 20 horas conseguir enviar los vídeos vía wetranfer. Todos estos obstáculos no sólo limitan la calidad sino también la cantidad de clases que puedo impartir y ensayos que puedo hacer por semana. Como consecuencia mi trabajo, tanto como profesora como bailarina, tuvo una decadencia. 


\section{Los vídeos de danza y vídeodanza}

Con la imposibilidad de representación en los teatros, las compañías, escuelas, alumnos y bailarines empezaron a poner vídeos de danza online y a producir vídeos de danza en casa y después en espacios al aire libre. La facilitad que el móvil y las plataformas trajeron para una simple edición y publicación hicieron de las redes sociales un nuevo escenario para la danza. Vídeos amateurs y profesionales, siendo éstos apenas un registro cinematográfico de coreografías creadas para bailar en vivo, o vídeos con coreografías pensadas para la cámara, grabadas en diferentes ángulos y editadas, que llamamos vídeodanza, estuvieron presentes en gran cantidad en internet durante la cuarentena. Bailarines, compañías de bharata natyam y kathak, y alumnos publicaron en las redes sociales los dos tipos de vídeos. Encontramos en mayor número registros cinematográficos de espectáculos producidos en el teatro, y registros de pequeñas coreografías y secuencias de movimientos hechas en casa, utilizadas tanto para divulgación del trabajo como para pasatiempo. En menor número encontramos los vídeodanza, presentes no sólo en las redes sociales sino además en festivales de danza online, que crecieron numerosamente durante la pandemia. ${ }^{8}$

Como ejemplos podemos citar la mayor plataforma de artes india del mundo, Indian Raga', que publica también vídeodanza en sus redes sociales y website, teniendo millones de visualizaciones en su canal en YouTube, y entre las danzas, el bharata natyam y el kathak con mayor presencia. El bailarín de kathak, Kumar

\footnotetext{
${ }^{8}$ Ejemplos de vídeos de danza y vídeodanza: https://www.youtube.com/watch?v=vayqpmFQhYU https://www.youtube.com/watch?v=sMbPilysleU https://www.instagram.com/tv/CIXFbhNATRP/ https://www.youtube.com/watch?v=v1lqr_x2HSM https://www.youtube.com/watch?V=SjhQV3f7qjY https://www.instagram.com/tv/CMTuzijh2-l/?utm_source=ig_web_copy_link

${ }^{9}$ https://indianraga.com/
} 
Sharma ${ }^{10}$, que ya tenía una presencia fuerte en Instagram pasó a publicar con más frecuencia vídeos de danza con coreografías grabadas dentro de casa alcanzando miles de espectadores. Abhinava Dance Company¹, compañía de bharata natyam y kathak, viene publicando vídeos de ensayos, de coreografías y de partes de sus espectáculos.

Otra característica de estos vídeos disponibles online es la duración. Las redes sociales ofrecen un número indeterminado de vídeos, el usuario está constantemente siendo bombardeado por información de todos los tipos, su atención está disipada en todo momento. Consecuentemente se pasó a producir vídeos cortos y que llamen la atención del usuario, para que éste sea visualizado hasta el fin.

Actualmente las personas tienen la necesidad de estar presentes en las redes sociales, compartir fotos, vídeos y momentos, y con la danza no es diferente. Los vídeos grabados por alumnos también crecieron, compartiendo en internet vídeos de clases y coreografías, enseñando al mundo lo que se está practicando dentro de casa.

\section{Acciones promovidas}

Pero más allá de transformar las clases presenciales en virtuales y producir vídeos, donde encontramos ejemplos innumerables, la pandemia generó diversas acciones que engloban la formación del pensamiento crítico y la formación de un profesional de la danza con capacidades no sólo técnica y artística sino también de negocios, liderazgo y marketing. Además dio la posibilidad de compartir conocimiento y experiencias con personas de varios países, posibilitó contenidos que antes no eran accesibles a nadie o a casi nadie, generando una red de colaboración en diferentes niveles y más democrática a nivel social y económico.

10 Bailarín, coreógrafo y cantor, hace diversos trabajos para la televisión india, coreografiando actrices reconocidas de bollywood, como Madhuri Dixit y Jacqueline Fernandez.

https://www.facebook.com/KumarSharmaDance

https://www.instagram.com/kumar1sharma/

${ }^{11}$ https://abhinava.dance/

https://www.instagram.com/nirupama_rajendra/ 
Las indias Mahalakshmi Prabhakar y Aranjani Bhargav crearon la plataforma Re-cognising dance ${ }^{12}$, un espacio democrático para dialogar, debatir y aprender sobre la historia de la danza india como también las cuestiones contemporáneas y actuales. A través de la página en Facebook divulgan sus eventos que se realizan en la plataforma Zoom, en los cuales cuentan con invitados para debatir algún temario en específico. Algunos vídeos se encuentran disponibles después en su canal de YouTube. Este espacio es muy importante para la concienciación sobre la historia y el proceso de reforma en las danzas indias durante el siglo pasado, incluyendo el bharata natyam y el kathak, así como cuestiones emergentes contemporáneas, que dialogan con cuestiones sociales y económicas, y sobre globalización e interculturalismo.

En Brasil fue creado en 2020 el foro A Dança Indiana no Brasill con la propuesta de promover un encuentro entre artistas, estudiantes, profesores y personas en general interesadas en debatir las diversas perspectivas de la danza india en Brasil de forma gratuita, vía plataforma Zoom. En una primera acción realizada en 3 encuentros en agosto de 2020, con duración de 3 horas cada uno, tuvo un total de 93 inscritos de todas las regiones del país, donde aproximadamente la mitad participó activamente de los debates. Este número a pesar de parecer pequeño, teniendo en consideración la poca difusión que existe en Brasil de la cultura india, es un número significativo. De estos encuentros surgió la necesidad de crear 3 subforos: pedagogía, historia e interculturalismo, donde cada persona pudo inscribirse en el subforo de su interés. Se celebraron de septiembre a diciembre de 2020 cada 15 días con una duración de 1:30h cada uno.

En 2021 el foro pasó a tener una acción mensual, siendo un encuentro para debatir un temario específico, intercalando con un directo en las redes sociales con un invitado para hablar sobre la historia de la danza india en Brasil. Además el foro cuenta con un blog donde hay testimonios, resúmenes de los 3 primeros encuentros, agenda de eventos organizada por los propios participantes y textos

\footnotetext{
12 https://www.mahalakshmiprabhakar.com/re-cognising-dance

https://www.facebook.com/recognisingdance

https://www.youtube.com/channel/UCCVPLrBWp6hQsPp3RHSkbCA

${ }^{13}$ https://dancaindianabrasil.wixsite.com/forum

https://www.facebook.com/fadinb2021

https://www.instagram.com/fadinb2021/
} 
de temáticas variadas. Un drive con una biblioteca virtual para consulta de los participantes. Perfiles en las redes sociales, Facebook, Instagram y YouTube para la divulgación de los eventos y de información. Y grupo en WhatsApp para comunicación entre los participantes y los organizadores.

Actualmente el foro sigue siendo gratuito e intenta crear un espacio horizontal de cambio de ideas, conocimientos y reflexiones, esperando contribuir para la formación de nuevos practicantes y pensantes de la danza india. Este movimiento está presente en otros países de la América Latina y del mundo, dialogando con las cuestiones históricas, pedagógicas e interculturales de cada país y continente.

Con la gran cantidad de contenido gratuito en internet, los profesionales de la danza también se encuentran con dificultad financiera generada con las crisis del COVID-19, en un primer momento donde muchos donaron conocimientos, esta acción pasó a ser inviable para aquellos que dependen del arte para sobrevivir, ya que los sectores de cultura y eventos son unos de los más perjudicados. Por esto las 4 organizadoras del foro crearon el Coletivo Trançado que busca a través de la enseñanza online un medio de adquirir recursos financieros. Ideado a finales de 2020 y puesto en práctica en el inicio de 2021 el colectivo ofreció en una primera acción un curso de formación titulado Dança Indiana na Cena Expandida compuesto de 5 talleres teóricos y prácticos impartido por las creadoras y por invitados, de marzo a julio de 2021, teniendo inscripciones de Brasil, México y Portugal. Adicionalmente fue creado un vídeo coreográfico pensado para la pantalla que buscó aproximar las culturas de los dos países, India y Brasil, utilizando la música brasileña Aflorou de Gustavito y Rafael Fares, con movimientos de las danzas indias y de danzas brasileñas, poniendo en práctica cuestiones interculturales debatidas en el foro. El vídeo fue divulgado en las redes sociales personales de las creadoras ${ }^{14}$.

En Argentina fue creado el Primer Congreso Argentino y Latinoamericano de Danzas Clásicas de la India ${ }^{15}$ realizado en los días 5, 6 y 7 de marzo de 2021,

${ }^{14}$ https://www.youtube.com/watch?v=LAPz8B8MxDM

15 https://www.gungurcongresos.com/1-congreso-danzas-cl\%C3\%A1sicas-de-la-in 
organizado y idealizado por Natalia Salgado, directora de Güngur Indian Fine Arts, con el apoyo de la Embajada de la India en Buenos Aires en la difusión en las redes y con subsidio del Ministerio de Cultura para pagar los invitados. El congreso ofreció gratuitamente conferencias, clases magistrales, mesas redondas, talleres, exposiciones, concursos y espectáculos online, brindando 18 ponencias. Los eventos especiales fueron la inauguración con el embajador de India, Dinesh Bhatia, el concurso literario, el foro para discutir sobre la danza clásica india insertada en latinoamérica, y el evento de cierre con danza en vivo. Y los invitados de honor fueron el maestro V.P, Dhananjayan ${ }^{16}$ (bharata natyam), Sujata Mohapatra $^{17}$ (odissi) y Sri Kishore Mosalikanti18 (Kuchipudi). El foro brasileño A Dança Indiana no Brasil recibió invitación para participar en el congreso y mediar el foro, teniendo una sección para exponer las acciones que se están llevando a cabo en Brasil y debatir las mismas cuestiones a nivel latinoamericano. Re-cognising también fue invitado a participar en el congreso con una sección para debatir la importancia de estudiar la historia de la danza, sobre el proceso de reforma del bharata natyam y sobre apropiación cultural. El congreso tuvo un total de 2100 inscripciones de Argentina, Brasil, Chile, Colombia, India y Perú.

En Europa fue creado el DDC - Digital Dance Collective por los artistas Amine Amourah (Francia), Anna Dimitratou (Grecia), Sneha Mistri (España) y Sumon Rudra (Grecia). El colectivo con el objetivo de apoyar a los artistas, aproximar a las personas, compartir y disfrutar los momentos, ofreció talleres prácticos de danza india. La 1a edición en marzo de 2020 así como la 8ạ en marzo de 2021 contó con 10 clases, los participantes hicieron donaciones del valor que pudieron. De la 2a edición a la 7a fueron ofrecidos 8 clases con el valor simbólico de 20 euros cada edición. Actualmente los organizadores están planeando la 9a edición para junio de 2021. Las clases impartidas vía grupo de Facebook contaron con un total de 68 clases y con 29 profesores de 12 países (Alemania, Canadá, España, Estados

\footnotetext{
${ }^{16}$ Vannadil Pudiyaveettil Dhananjayan y su esposa Shanta Dhananjayan estudiaron en Kalakshetra, siguiendo una carrera juntos como bailarines y profesores, actuando en varias partes del mundo y recibiendo diversos premios.

17 Sujata Mohapatra es una de las bailarinas más conocidas de su generación en India y en el exterior, se dedicando principalmente a la enseñanza, también ha recibido diversos premios.

18 Kishore Mosalikanti es bailarín, profesor y coreógrafo, aclamado por su técnica perfecta y por las innovaciones en sus composiciones.
} 
Unidos, Finlandia, Francia, Grecia, India, Inglaterra, Italia, Polonia y Singapur). Participaron de la 2a a la 7a edición una media de 100 alumnos cada una, de varias partes del mundo; la 1a y la 8a edición por haber sido abierto al público, no se sabe cuántas personas participaron pero el grupo en Facebook tiene $2.149^{19}$ integrantes.

En este colectivo el foco es la danza bollywood, que es el baile del cine indio que mezcla varios tipos de danza, orientales y occidentales, dependiendo de la canción y del contexto de la película. Los talleres con coreografías influenciadas por danza clásica india fueron del kathak, que llaman de semiclásico, pasos de danza clásica con algunas variaciones e insertados en una música no clásica, esto es, de bollywood. Uno de los profesores a impartir estos talleres fue Kumar Sharma, citado anteriormente. Estas coreografías tienen una aceptación mayor del público ya que son menos complejas que las coreografías clásicas, alcanzando un número mayor de practicantes.

La Kalakshetra ${ }^{20}$, escuela fundada por Rukmine Devi Arundale, a pesar de tener website y perfiles sociales, no hacía publicaciones ni promovía eventos en estos medios, sólo en 2020 con la pandemia del COVID-19 que empezó a estar presente en el mundo virtual promoviendo clases, conferencias y poniendo en YouTube sus espectáculos por tiempo limitado. En 2021 estos eventos y vídeos online disminuyeron considerablemente.

Empresas de consultorías para artistas, como por ejemplo, Shreya Nagaraja Singh ${ }^{21}$ y Aalaap $^{22}$ pasaron a estar presentes con más frecuencia en las redes sociales promoviendo conferencias, cursos, representaciones gratuitas y pagadas. Ayudando artistas a desarrollar sus carreras profesionales, tanto en lo artístico como en lo financiero. Shreya Nagaraja Singh viene presentando nuevas formas y

\footnotetext{
19 Información accedida en: 22 abr. 2021 a las 17:22.

20 https://www.kalakshetra.in/newsite/

https://www.facebook.com/profile.php?id=100007605438575

https://www.instagram.com/kalakshetrafoundation/

${ }^{21}$ https://www.shreyanagarajansingh.com/

https://www.facebook.com/shreyanagarajan1

https://www.instagram.com/p/B-AOtYQDloG/

22 http://www.theaalaap.com/

https://www.facebook.com/theaalaap

https://www.instagram.com/aalaap_concepts/
} 
estrategias de producir arte y de poner valor en él, planeando de manera integral la carrera del artista y del bailarín. Creó los eventos What happens next? y For the future que tuvo varias ediciones con diversos invitados de diferentes áreas: negocios, finanzas, subsidios, entrenamiento e investigación artística, entre otras.

La artista independiente Veena Basavarajaiah ${ }^{23}$ tiene en Instagram la página Cartoon natyam donde a través de dibujos hace críticas y cuestiona variadas temáticas que involucran estereotipos, tabús, tradición y la danza india. Sus publicaciones representan alumnos y profesores/maestros de bharata natyam, sus familiares y el público en general. Durante la cuarentena de 2020 sus publicaciones aumentaron, estando más presente en la red social y levantando más cuestiones sobre la preservación, la ética, los valores y la incoherencia de la tradición.

\section{Consideraciones finales}

A través de los ejemplos citados, y de lo que está ocurriendo desde el inicio de la pandemia del COVID-19 hasta el momento presente podemos observar que la transformación de la danza para el medio digital ocurrió por necesidad y sin una preparación previa. Las clases online al mismo tiempo que dan la oportunidad de tener clases con otros profesores, de otras ciudades y países, limitan el proceso de enseñanza-aprendizaje, dependiendo de la de calidad de la plataforma usada, de la conexión a internet y del espacio disponible para la práctica, como también el propio vehículo digital limita este proceso, ya que lo convierte en bidimensional y la danza precisa de la tercera dimensión para su total comprensión, principalmente para alumnos principiantes.

A pesar de estas limitaciones, el uso de la tecnología vino como una estrategia momentánea que está siendo desarrollada y testada por los profesionales, abriendo un nuevo mercado para la danza, principalmente para promover la

23 Veena Basavarajaiah es una artista contemporánea que trabajó con reconocidas compañías en India e Inglaterra, actualmente trabaja independientemente colaborando con otros artistas y compañías.

http://www.veenadance.com/

https://www.facebook.com/veenadance

https://www.instagram.com/cartoon_natyam/ 
enseñanza a través del mundo, conectando en un mismo aula virtual personas de varios lugares. Probablemente ésta será una herramienta permanente y mejorada en la danza.

La pandemia también impulsó la creación de eventos, foros y congresos online, conectando personas y estableciendo lugares para reflexión crítica sobre la teoría, la práctica y la profesionalización de la danza, contextualizando las danzas clásicas indias, bharata natyam y kathak, en el mundo actual, globalizado y digital.

La creación/producción/recepción pasó a ser generada para la pantalla, siendo por vídeodanza o por vídeos de danza. Los vídeos de danza sólo grabados son los que encontramos en mayor número, secuencias de movimientos y coreografías hechas en casa y en espacios libres, tanto para divulgar el trabajo del profesional como también del alumno para marcar su presencia en las redes sociales. Influyen también en la recepción, donde el público recibe infinitas opciones y dispone de un tiempo limitado de atención, dando preferencia a vídeos cortos e impactantes.

Comparando los 6 D's Exponencial de Peter Diamantis con la danza india, podemos percibir que, la danza fue digitalizada, pasó a estar presente en el mundo virtual de diversas maneras (1 D) no siempre de la manera ideal ya que la enseñanza encontró las dificultades apuntadas anteriormente, y los artistas también tuvieron dificultad en saber cómo existir en el medio digital, provocando una decepción (2 D). Este cambio generó una disrupción (3 D), la danza india salió de su medio para un nuevo ambiente digitalizado. La desmonetización (4 D) fue usada a principio pero se convirtió en inviable, teniendo los artistas que crear una nueva forma de monetizar para poder sobrevivir, como por ejemplo, el Coletivo Trançado, en Brasil, y el Digital Dance Collective, en Europa. La desmaterialización (5 D) materializó, paradójicamente, algo que es inmaterial, la danza existe en el momento presente, cuando las luces del teatro se apagan, la danza deja de existir, pero en el medio digital ella permanece, materializada en diversos vídeos de danza y vídeodanza, además de las clases que son grabadas. La tecnología posibilitó la democratización (6 6 D) de la danza, pero podemos percibir que esta democratización no es accesible a todos, ya que no basta que un contenido sea 
gratuito en internet, otros factores están involucrados, como espacio disponible para la práctica, calidad de la plataforma ofrecida, calidad del aparato utilizado, calidad de la conexión a internet, esto si hay internet. Democratizar en sentido amplio, tanto la danza como cualquier otra área de conocimiento, producto o servicio aún está lejos de acontecer, es un proceso en construcción.

Como podemos ver, varias acciones fueron y continúan siendo realizadas en tiempos pandémicos. Además de transformar las clases presenciales en remotas, la crisis sanitaria creó un nuevo campo para proporcionar una nueva formación al bailarín, que engloba una nueva manera de ver y entender la profesión, pensando y reflexionando la práctica y la teoría de la danza india de forma crítica y constructiva, uniendo saberes y personas por todo el mundo en la tentativa no sólo de continuar existiendo sino de crear nuevas oportunidades de trabajo y de aumentar la calidad de lo que se enseña, de lo que se aprende, de lo que se divulga, de lo que se transmite.

La danza clásica india, el bharata natyam y el kathak, pasaron del templo, a los palacios, a las calles, a los teatros y al mundo digital, se desarrollaron y cambiaron la técnica, la forma estética, el proceso de aprendizaje-enseñanza, la representación, la creación, la producción, la recepción en el transcurso de los años. Las nuevas tecnologías, la digitalización de la danza, el tiempo pandémico en que vivimos, es solamente una fase en la historia de estas danzas que entre aciertos y errores busca nuevos caminos para seguir adelante, conectando tradición y tecnología, como una nueva modalidad de transmisión de saberes milenarios. El camino aún es largo pero la pandemia aceleró un proceso que venía ocurriendo y probablemente la danza india continuará en el medio digital encontrando nuevas maneras de existir.

\section{Referências}

BERNAL, Óscar Cornago. O corpo invisível: teatro e 20magen20gías da 20magen. Urdimento - Revista de Estudos em Artes Cênicas, Florianópolis, v. 2, n. 11, p. 177-189, 2008. 
KERSENBOOM, Saskia C. Nityasumangali. New Delhi: Motilal Banarsidass, 1987.

LACHINO, Haydé. Danza y tecnología. In: Adriana Guzmán (Org.) México coreográfico. Danzantes de letras y pies. Ciudad de México: Instituto Nacional de Bellas Artes y Secretaría de Cultura, 2017, p. 271-280.

LAMAS-BAIAK, Miriam. La danza clásica india bharata natyam y su relación con el ballet clásico occidental en los siglos XIX y XX. 2019. TFM (Trabajo de conclusión de curso) (Máster en Artes Escénicas) - Instituto de Danza Alicia Alonso Universidad Rey Juan Carlos, Madrid, España, 2019.

LÓPEZ, Rubén D. Alves. Las TIC y su efecto catalizador en los itinerarios de enseñanza y aprendizaje: plataforma black board collaborate. In: María Elena del Valle Mejías (Org.) Tecnoaulas y nuevos lenguajes educativos. España: Tecnos, 2018, p. $35-45$.

KOTHARI, Sunil. Kathak: indian classical dance art. New Delhi: Abhinav Publications, 1989.

MEDURI, Avanthi. The transfiguración of indian/asian dance in the United Kingdom: contemporary bharatanatyam global contexts. Asian theatre journal, v. 25, n. 2, p. 298 - 328, 2008.

MATTOS, Flávia. Entrevista realizada por el autor a través de un cuestionario con preguntas abiertas enviado por correo electrónico en el día 25 de abril de 2021 y recibido en 04 de mayo de 2021.

MEREJO, Andrés. La globalización del cibermundo. Trilogía Ciencia Tecnología Sociedad, Medellín - Colombia, v.9, n.17, p. 175-187, 2017.

MUNI, Bharata. The natya sastra. Nueva Delhi, India: Sri Satguru, 2006.

NACHIAPPAN, C. (Org.). Rukmini Devi: Bharata natya. Chennai: Kalakshetra Publications, 2001.

NARAYAN, Shovana. Kathak: Rhytmic echoes and reflections. New Delhi: Roli Books, 2001.

NARAYAN, Shovana. Kathak. New Delhi: Wisdom Tree, 2004.

NARAYAN, Shovana. Indian classical dances. New Delhi: Shubhi Publications, 2005.

SKIBA, Katarzyna. Cultural geography of kathak dance: Streams of tradition and global flows. In: Cristina Bignami e Ewa Debicka-Borek (Org.) Cracow indological studies, vol. XVIII. Kraków: Ksiegamia Akademicka, 2016, p. 55-91.

TAVARES, Fernando Gomes de Oliveira. O conceito de inovação em educação: uma revisão necessária. Revista do Centro de Educação. UFSM, 44, p. 1-19, 2019. 
TREDEZINI, Bárbara. Entrevista realizada por el autor a través de un cuestionario con preguntas abiertas enviado por correo electrónico en el día 24 de septiembre de 2020 y recibido en 12 de noviembre de 2020.

WALKER, Margaret. Kathak dance: a critical history. 2004. Tese (Doctorado en Filosofía) - University of Toronto, Canadá, 2004.

WALKER, Margaret. Kathak log ya kathak nrtya: The search for a dance called kathak. Journal of the indian musicological society, India, 2009, n.40, p. 168-190.

WALKER, Margaret. Courtesans and choreographers: The (re)placement of woman in the history of kathak dance. In: Pallabi Chakravorty y Nilanjana Gupta (Org.) In: dance matters: Performing india. New Delhi: Routledge, 2010a, p. 279-300.

WALKER, Margaret. Revival and reinvention in India's kathak dance. MusicCultures, Canadá, n.37, 171-184, 2010b.

Recebido em: 07/06/2021

Aprovado em: 19/08/2021 